\title{
Research status and hotspots of cesarean scar pregnancy from 2001 to 2020: a bibliometric analysis
}

\author{
Pin Qiu ${ }^{1}$, Xinyi Lin ${ }^{2}$, An Li $^{1}$, Gaopi Deng ${ }^{1}$ \\ ${ }^{1}$ Department of Gynaecology, The First Affiliated Hospital of Guangzhou University of Chinese Medicine, Guangzhou, China; ${ }^{2}$ First Clinical \\ Medical School, Guangzhou University of Chinese Medicine, Guangzhou, China \\ Contributions: (I) Conception and design: P Qiu, G Deng; (II) Administrative support: G Deng; (III) Provision of study materials or patients: P Qiu, \\ G Deng; (IV) Collection and assembly of data: P Qiu, X Lin, A Li; (V) Data analysis and interpretation: P Qiu, X Lin, A Li; (VI) Manuscript writing: \\ All authors; (VII) Final approval of manuscript: All authors. \\ Correspondence to: Gaopi Deng. Department of Gynaecology, The First Affiliated Hospital of Guangzhou University of Chinese Medicine, Jichang \\ Road 16\#, District Baiyun, Guangzhou 510405, China. Email: denggaopi@126.com.
}

Background Due to the significant increase in cesarean section rates worldwide, cesarean scar pregnancy (CSP) has attracted extensive attention. This study aimed to investigate the global research status and developmental directions of CSP to discern promising research hotspots by means of bibliometrics and visualized analyses.

Methods: The English CSP-related literature from 2001 to 2020 was retrieved from The Web of Science Core Collection (WOSCC) database. Microsoft Excel 2019 was used to analyze the years, countries, institutions, journals, authors, citations, $\mathrm{H}$ indices, titles, abstracts, and keywords of related literature, and VOSviewer software was used to visualize developmental directions and promising hotspots in CSP.

Results: The study included 1,186 articles in total. The number of CSP articles has presented an overall increasing trend over the last two decades. China has the maximum number of publications, but America's $\mathrm{H}$-index is higher than that of China, with the average number of citations per item ranking fourth. The Fournal of Obstetrics and Gynaecology Research published the most on the subject, while Zhejiang University from China published the largest number of articles; Jurkovic D, Timor-Tritsch IE, and Monteagudo A have achieved considerable progress in the CSP domain. There are 5 clusters that the current research orientation can fall into: "selection of the delivery mode with a scarred uterus after cesarean section", "risk factors", "diagnosis", "treatment" and "related basic and clinical experiments". High-intensity focused ultrasound (HIFU) and the formulation of consensus guidelines are the focus of the current research.

Conclusions: The analysis of global trends shows that CSP research is being actively studied and that the number of published studies is continuously increasing. According to the comprehensive quality and quantity of the literature, the United States maintains a leading position in CSP research. In recent years, the research directions have mainly focused on "diagnosis", "treatment" and "risk factors", while HIFU and the formulation of consensus guidelines may become research hotspots in the field of CSP.

Keywords: Bibliometric analysis; cesarean scar pregnancy (CSP); research status; hotspots; VOSviewer

Submitted Dec 03, 2021. Accepted for publication Feb 11, 2022.

doi: 10.21037/apm-21-3976

View this article at: https://dx.doi.org/10.21037/apm-21-3976 


\section{Introduction}

Cesarean scar pregnancy (CSP) refers to a zygote implanted in the scar site of a previous cesarean section (1), which can cause severe consequences such as massive bleeding, uterine rupture, and even hysterectomy as a specific type of ectopic pregnancy (2) and may affect the quality of life in the long term (3).

CSP was reported by Larsen and Solomon for the first time in 1978. Its incidence has been estimated to range from 1:1,800 to 1:2,216 (4). As cesarean delivery has soared worldwide in the past two decades, a general trend of increasing CSP has emerged because it is one of the longterm complications of cesarean delivery (5). The cesarean delivery rate in comprehensive hospitals in mainland China has grown to $41.1 \%$ (6), and the rate of scarred uteri in pregnant women is up to $17.7 \%$ (7). CSP occurs in approximately $5 \%$ of women diagnosed with ectopic pregnancy who have had a previous cesarean section (8).

To date, the pathogenesis of CSP remains unclear. Most scholars hold the view that CSP involves defects that heal after interruptions in the continuity between the myometrium and endometrium at the site of incision after cesarean section. It has been reported that maternal age, parity, hospital grade with respect to previous uterine surgeries, the interval between the last cesarean section and the current pregnancy, the number of induced abortions (especially after cesarean delivery), and the thickness of the lower uterine segment can act as risk factors for CSP by affecting the healing of uterine incision in multiple ways (9). Benefiting from the progress of diagnostic techniques, especially the development of transvaginal ultrasound, the sensitivity and precision of CSP diagnosis have relatively increased. However, due to the diversity of symptoms, CSP remains difficult to diagnose, and several atypical cases may be misdiagnosed as cervical pregnancy, trophoblastic tumor and inevitable abortion. To date, a multitude of treatment regimens have been proposed, including pharmacotherapy, uterine artery embolization (UAE), ultrasound-guided curettage, high-intensity focused ultrasound (HIFU), nidus excision and hysterectomy. Whether these therapies can improve subsequent pregnancy outcomes and reduce the incidence of recurrent CSP (RCSP) has not yet been definitively confirmed, and the optimal treatment therefore remains to be determined. Obstetricians and gynecologists should provide individualized therapy regimens based on a combination of patients' conditions and medical management.
Recently, CSP has attracted extensive interest, and a growing number of domestic and overseas studies of CSP have been conducted. However, only limited information on the research status and hotspots of CSP has been reported at present. Bibliometrics, as a tool used to measure the contributions of authors, journals, institutions and countries in a specific research field, can also qualitatively and quantitatively evaluate global research trends to guide future research directions. This technique has been widely used in numerous medical research areas in recent years (10-12). Zhang has conducted the first bibliometric analysis of CSP (13) to our knowledge, while the study focused less on the coupling analysis which contributes to reveal underlying cooperative relationships among authors, countries or institutions and was lack in temporal dynamics of evolving hotspots which can mine the research frontier of CSP. In this study, we applied bibliometrics and visualized analysis to analyze and summarize the global research status of CSP including its developmental trend, literature quantity and quality assessment, global cooperative relationships and hotspots development dynamics to provide a reference for obstetricians and gynecologists to grasp research directions and hotspots in this field and explore cutting-edge management.

\section{Methods}

\section{Data retrieval strategy}

We used the Web of Science Core Collection (WOSCC), the most widely used and appropriate database for bibliometric analysis, and we searched and obtained the global literature on CSP from January 2001 to January 8, 2021 (the last 20 years). We collected all the records retrieved from the literature, downloaded and saved them in ".txt" format, and imported them into Microsoft Excel 2019 for analysis. The search strategy applied to data retrieval was as follows: "TS $=$ (cesarean scar pregnancy or cesarean scar pregnancy or cesarean scar pregnancies or scar pregnancy or scar pregnancy after cesarean section or cesarean scar ectopic pregnancy or cesarean uterine scar pregnancy) AND publishing year $=(2001-2020)$ AND language $=($ English $)$ AND Document types $=($ ARTICLE $) "$

\section{Statistical analysis}

After retrieving the results, we clicked the option of "analyze search results" on the website and then clicked the 


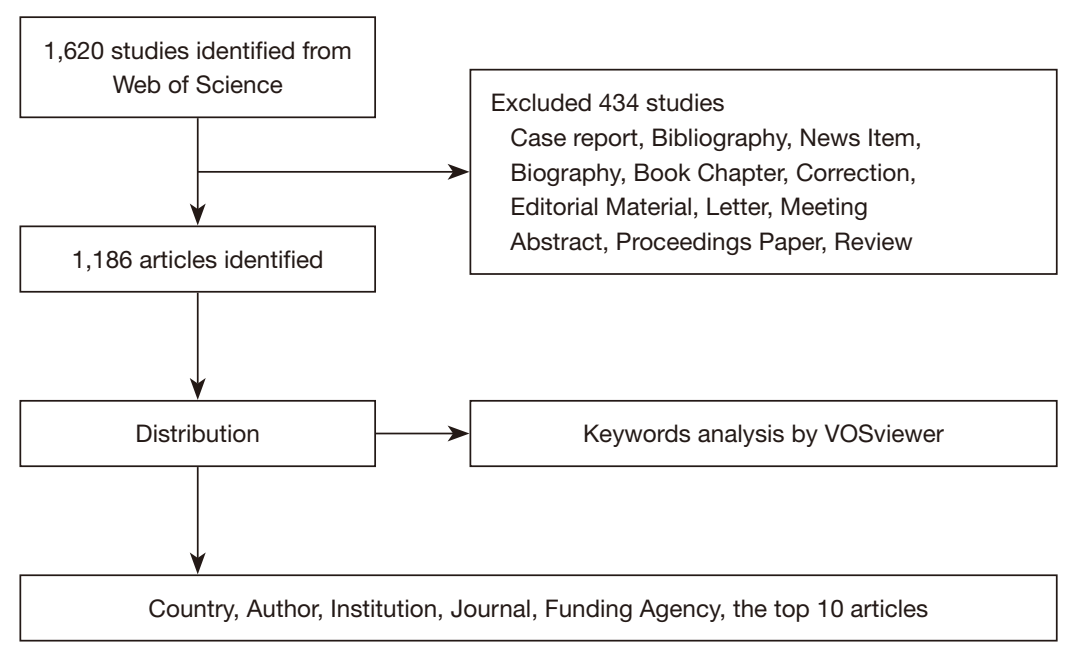

Figure 1 Flow chart of retrieval and analysis.

top 20 countries successively to "create citation reports". The total citation frequency and $\mathrm{H}$ index of each country were imported and analyzed in Microsoft Excel 2019. The citation frequency refers to the total number of cited references in the corresponding years, which reflects the level of acceptability of an article among scholars. The $\mathrm{H}$ index is defined as "a journal or institution has $\mathrm{n}$ of its articles each cited at least $\mathrm{n}$ times" and reflects the quantity and quality of literature published by researchers and institutions (14). We analyzed the basic data characteristics of the abovementioned documents in the database, used the drawing function in Excel software to analyze the obtained ".xls" format files, and generated the corresponding data distribution map.

VOSviewer (Leiden University, Netherlands) has been widely applied to construct and visualize bibliometric networks. We imported all the downloaded TXT files into VOSviewer 1.6.15 to carry out literature cocitation analysis and keyword co-occurrence analysis.

Bibliographic coupling, which is helpful for identifying similarities between articles by citation analysis, was used to identify correlations among authors, institutions, countries and regions. The number of circles in the figure indicates the number of analyzed items, and a larger circle corresponds to a greater contribution from the analyzed item. The line between circles represents the correlation between the analyzed items, and a thicker or longer line corresponds to a greater correlation. Keyword cooccurrence analysis is used to grasp the current research status and explore promising hotspots in this field. In the visualized analysis diagram, the number of circles in the figure indicates the number of analyzed items. The size of the nodes indicates the frequency of occurrence of a keyword; that is, a larger point corresponds to a greater likelihood of becoming a hotspot in the research fields of CSP.

\section{Results}

\section{Global research publications and projections}

A total of 1,620 studies related to CSP between 2001 and 2020 were obtained from the Web of Science. Among these, 1,186 publications met the inclusion criteria (Figure 1). As shown in Figure 2A, although the number of relevant studies has fluctuated slightly over the past two decades, an overall upward tendency is evident, and publications in 2020 hit the peak (126 papers). According to the curve model, the number of publications can be predicted to increase to nearly 200 by 2025 .

\section{Distributions and contributions of countries and regions}

Related studies have been carried out in 80 countries (Figure 2B). The number of articles written in Chinese ranked at the top (267 papers accounting for $22.513 \%$ ), followed by the USA (230 papers accounting for $19.393 \%$ ) and England ( 74 papers accounting for $6.239 \%$ ). Publications of the top three countries accounted for approximately half of the total. The period from 2012 to 2020 witnessed a slight increase in articles published in 

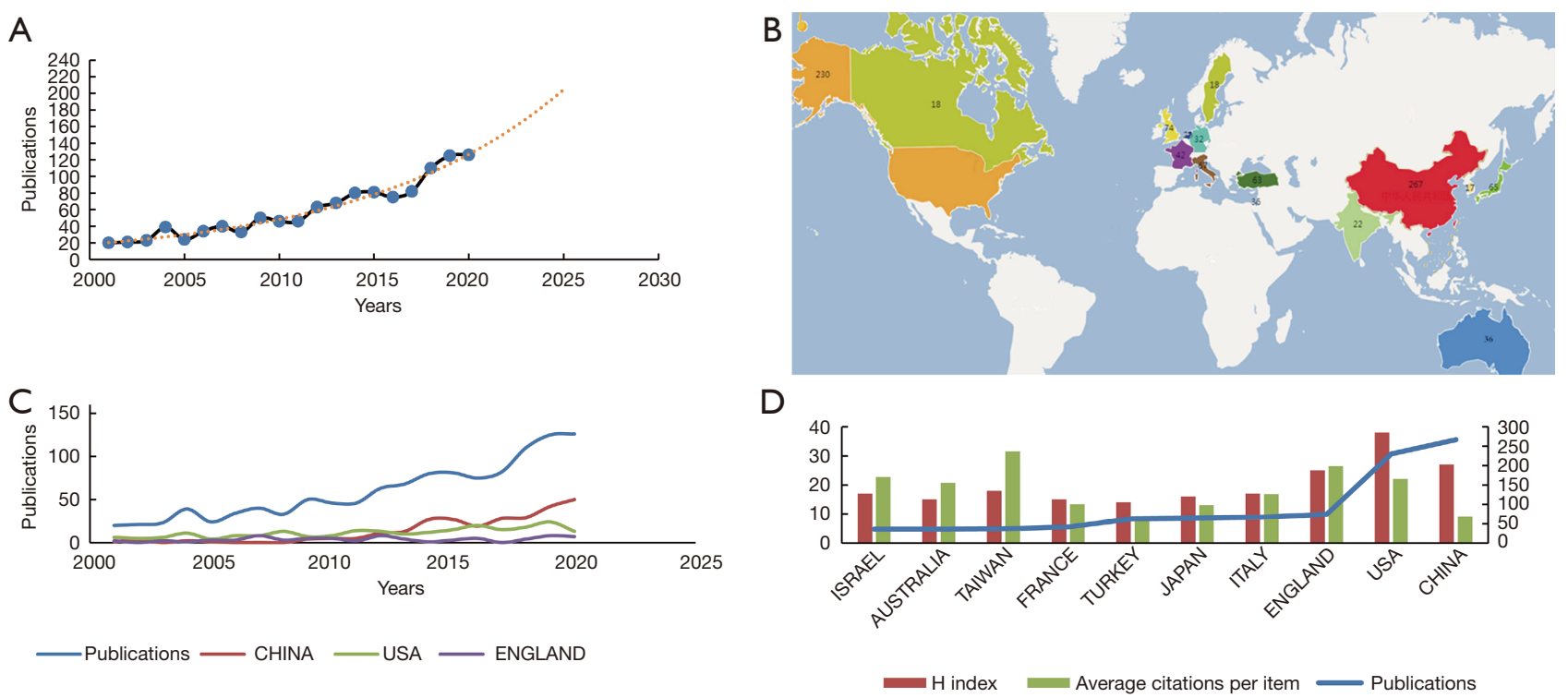

Figure 2 Tendency and distribution of global research publications. (A) Curves: growth trends of the cumulative number of publications on CSP worldwide from 2001 to 2020. (B) Distribution of CSP research in the world map. (C) The number of publications from China, the USA and England over a 20-year period. (D) The total number of articles published, the $\mathrm{H}$ index and the average number of citations per article in the top 10 countries or regions. CSP, cesarean scar pregnancy.

China and the USA. China reached its highest point at 50 papers in 2020 (Figure 2C). However, in terms of the $\mathrm{H}$ index, the USA [38] overtook China [27] and England [25], being second to none. By comparing the average number of citations per item, we found that Taiwan ranked first (31.51) and England ranked second (26.45), followed by Israel (22.69) and the USA (22.08) with almost the same numbers (Figure 2D).

\section{Authors with the bighest contributions to CSP}

Timor-Tritsch IE (17 papers, H index $=11$ ) and Monteagudo A (16 papers, $H$ index $=11)$ are the top two authors who have contributed the most articles with an almost equal number of publications and the same $\mathrm{H}$ index. However, in contrast to Jurkovic D, whose articles were cited the most (74.27), Timor-Tritsch IE (35.53) and Monteagudo A (37.06) had significantly lower citation frequencies (Figure $3 A$ ).

\section{Institutions with the highest contributions to CSP}

Figure $3 B$ depicts information about the top ten institutions with the highest contributions, which shows that Zhejiang University from China published the most articles (26 papers), followed by New York University (NYU) (17 papers) from the USA and Shanghai Jiao Tong University (17 papers) from China. NYU had the highest H index [12], followed by King's College Hospital London (Kings Coll Hosp London) [11] from England and Zhejiang University [10]. For the average number of citations per item, Kings Coll Hosp London ranked first (65.73), NYU ranked second (38.76), and Tel Aviv University ranked third (22.13), while Zhejiang University (11.15) and Shanghai Jiao Tong University (7.82) seemed inferior.

\section{Fournals with the highest contributions to CSP}

As shown in Figure 3C, Fournal of Obstetrics and Gynaecology Research published the most articles [55], followed by Ultrasound in Obstetrics Gynecology [41] and American Fournal of Obstetrics and Gynecology [38]. Nevertheless, Ultrasound in Obstetrics Gynecology ranked first for the average citation frequency (49.73) and $\mathrm{H}$ index [23], followed by American Fournal of Obstetrics and Gynecology (45.79 citations, $\mathrm{H}$ index $=20$ ), which far exceeded Fournal of Obstetrics and Gynaecology Research (7.76 citations, $\mathrm{H}$ index $=12$ ).

\section{Funding agency analysis}

Of the top ten relevant funding agencies (Table 1), four are 

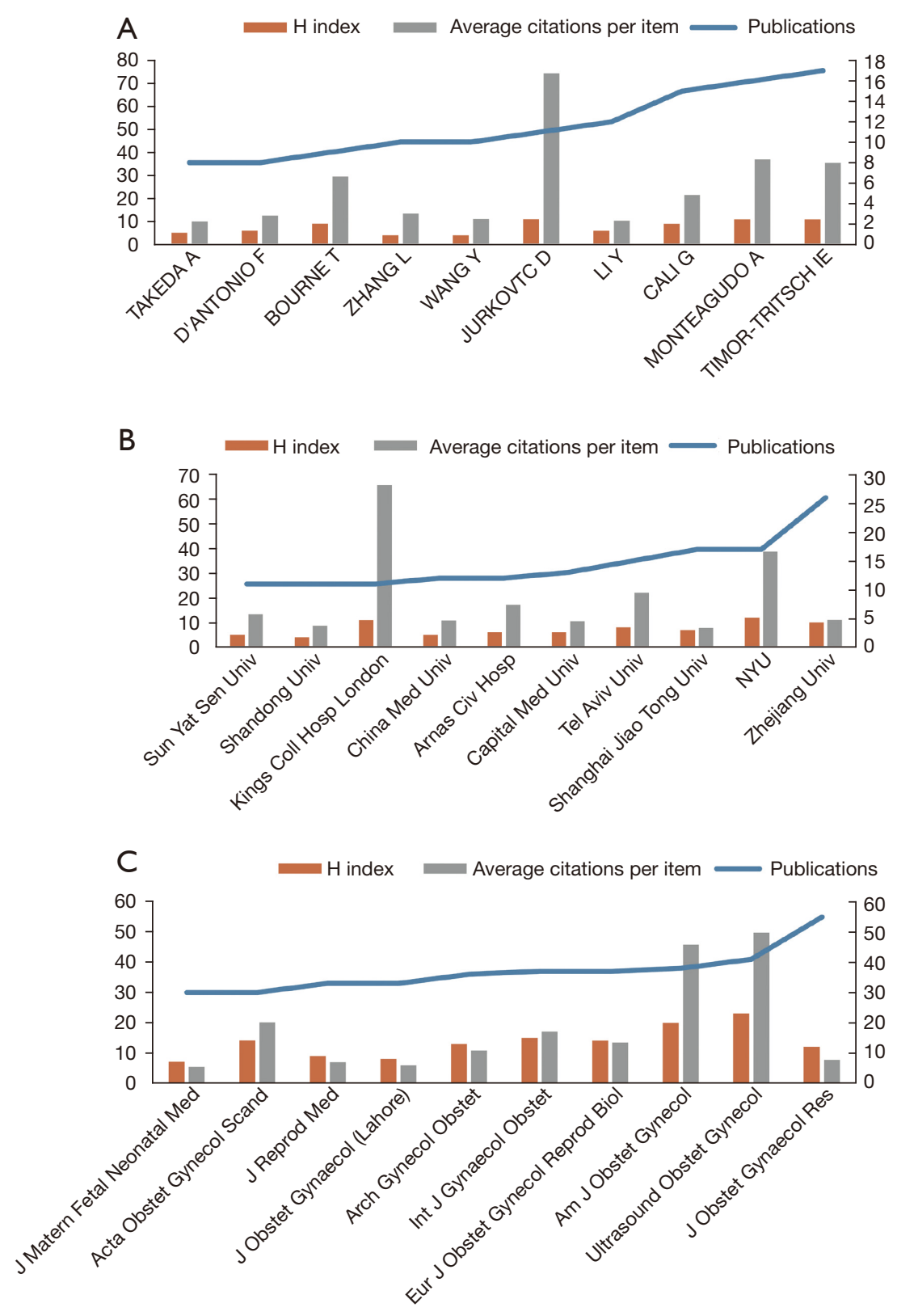

Figure 3 A composite chart of the total publications, H index and average number citations per article. (A) Regarding authors; (B) regarding institutions; (C) regarding journals. Sun Yat Sen Univ, Sun Yat-sen University; Shandong Univ, Shandong University; Kings Coll Hosp London, King's College Hospital London; China Med Univ, China Medical University; Arnas Civ Hosp, Arnas Civic Hospital; Capital Med Univ, Capital Medical University; Tel Aviv Univ, Tel Aviv University; Shanghai Jiao Tong Univ, Shanghai Jiao Tong University; NYU, New York University; Zhejiang Univ, Zhejiang University; 7 Matern Fetal Neonatal Med, Fournal of Maternal Fetal Neonatal Medicine; Acta Obstet Gynecol Scand, Acta Obstetricia Et Gynecologica Scandinavica; 7 Reprod Med, Fournal of Reproductive Medicine; 7 Obstet Gynaecol (Lahore), Fournal of Obstetrics and Gynaecology; Arch Gynecol Obstet, Archives of Gynecology and Obstetrics; Int 7 Gynaecol Obstet, International fournal of Gynecology Obstetrics; Eur 7 Obstet Gynecol Reprod Biol, European Fournal of Obstetrics Gynecology and Reproductive Biology; Am 7 Obstet Gynecol, American Journal of Obstetrics and Gynecology; Ultrasound Obstet Gynecol, Ultrasound in Obstetrics Gynecology; 7 Obstet Gynaecol Res, Fournal of Obstetrics and Gynaecology Research. 
Table 1 The top 10 related funding agencies

\begin{tabular}{lcc}
\hline Funding agency & Number \\
\hline National Institutes of Health NIH USA & 40 & 3.373 \\
United States Department of Health Human Services & 40 & 3.373 \\
National Natural Science Foundation of China NSFC & 30 & 17 \\
NIH Eunice Kennedy Shriver National Institute of Child Health Human Development NICHD & 11 \\
Ministry of Education Culture Sports Science and Technology Japan MEXT & 10 \\
NIH National Institute of Allergy Infectious Diseases NIAID & 9.530 \\
Japan Society for the Promotion of Science & 7.433 \\
Grants in Aid for Scientific Research KAKENHI & 0.927 \\
Medical Research Council UK MRC & 7 \\
Wellcome Trust & 7 & 0.759 \\
\hline
\end{tabular}

from the USA, three are from Japan, two are from the UK, and one is from China. National Institutes of Health NIH USA and United States Department of Health Human Services from the USA had an equal number of publications (40 articles).

\section{Characteristics of the top ten CSP articles}

Table 2 demonstrates the situation of the top 10 cited research papers in terms of topic, first author, year, journal, impact factor, citations and major conclusion. Of the ten papers, four were published in Ultrasound in Obstetrics \& Gynecology, and three were published in American Fournal of Obstetrics and Gynecology. "First-trimester diagnosis and management of pregnancies implanted into the lower uterine segment cesarean section scar", written by Jurkovic $\mathrm{D}$ and published in Ultrasound in Obstetrics \& Gynecology in 2003, was cited the most frequently [386].

\section{Coupling analysis}

Bibliographic coupling is helpful for identifying similarities between articles by citation analysis. The number of circles in the figure represents the number of items, and a larger circle corresponds to a greater contribution from the analyzed item. The line between circles indicates the correlation between the analyzed items, where a thicker or longer line corresponds to a greater correlation.

A total of 5,482 authors were included in the published literature, and 26 authors (defined as each author with at least 5 related studies) were analyzed using VOSviewer
(Figure 4A). The top five authors with high total coupling strength were as follows: Timor-Tritsch IE (total coupling strength $=3,601$ ), Monteagudo A (total coupling strength $=3,536$ ), Cali Giuseppe (total coupling strength $=2,353$ ), Cali G (total coupling strength $=2,352$ ) and Agten AK (total coupling strength $=2,167$ ).

The study enrolled 1,465 institutions, and 66 institutions (defined as each institution with at least 5 studies) were analyzed using VOSviewer (Figure 4B). The top five institutions with high total coupling strength were as follows: NYU (total coupling strength $=7,058$ ), Zhejiang University (total coupling strength $=7,053$ ), Arnas Civic Hospital (total coupling strength $=4,734$ ), Shanghai Jiao Tong University (total coupling strength $=4,715$ ), and Tel Aviv University (total coupling strength $=4,284$ ).

Eighty countries were included in the study, and 39 countries (defined as each country with at least 5 studies) were analyzed using VOSviewer (Figure 4C). The top five countries with large total coupling strength were listed below: China (total coupling strength $=94,652$ ), the USA (total coupling strength $=57,204$ ), England (total coupling strength $=31,272)$, Turkey (total coupling strength $=26,358$ ), and Italy (total coupling strength $=20,941$ ).

\section{Hotspot analysis of CSP}

The co-occurrence analysis of keywords by VOSviewer was used to identify the present hotspots and explore the future hot areas of CSP research. Node size denotes the frequency of occurrence of the keywords; that is, a larger point corresponds to a greater likelihood of becoming a hotspot 


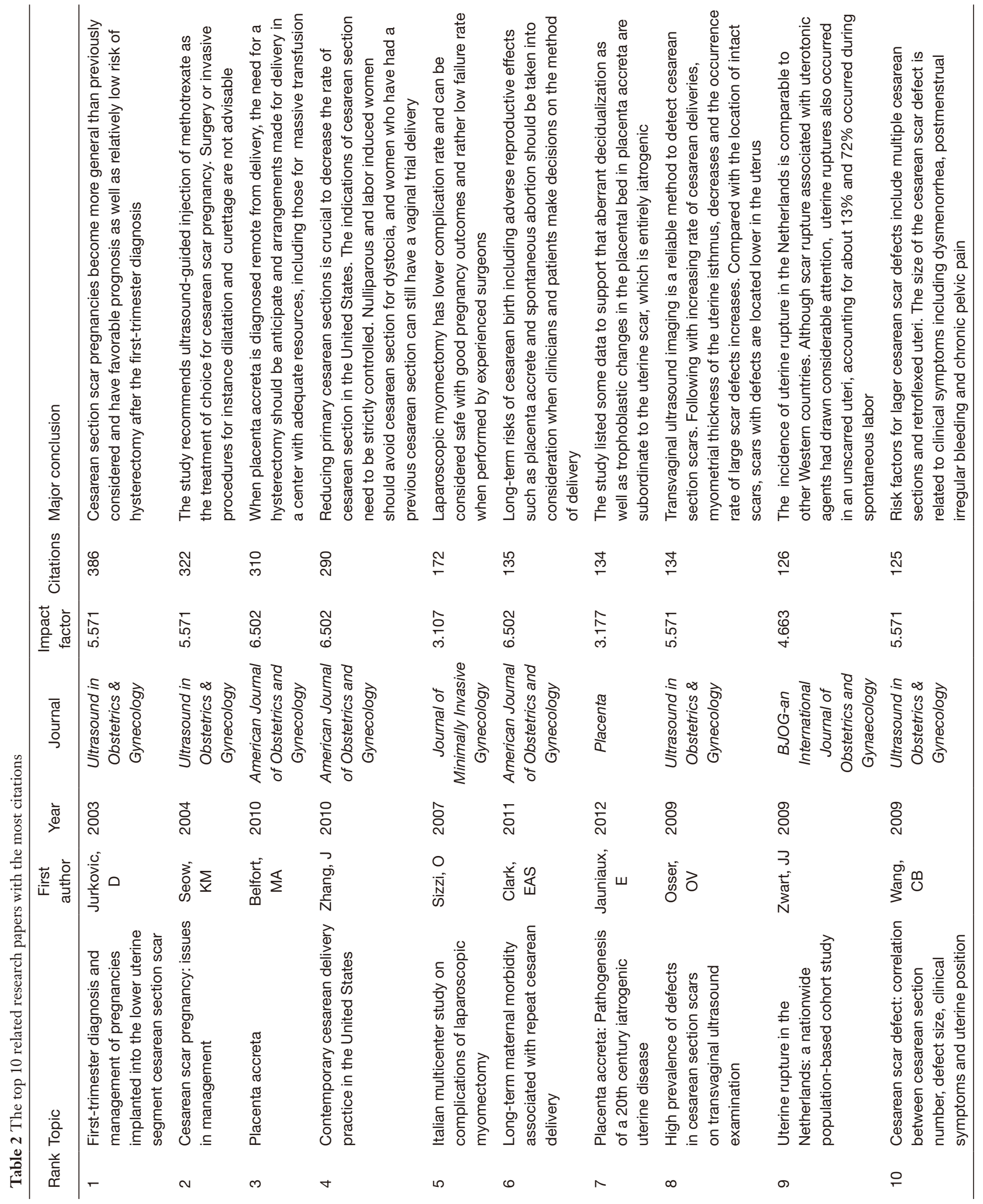


A

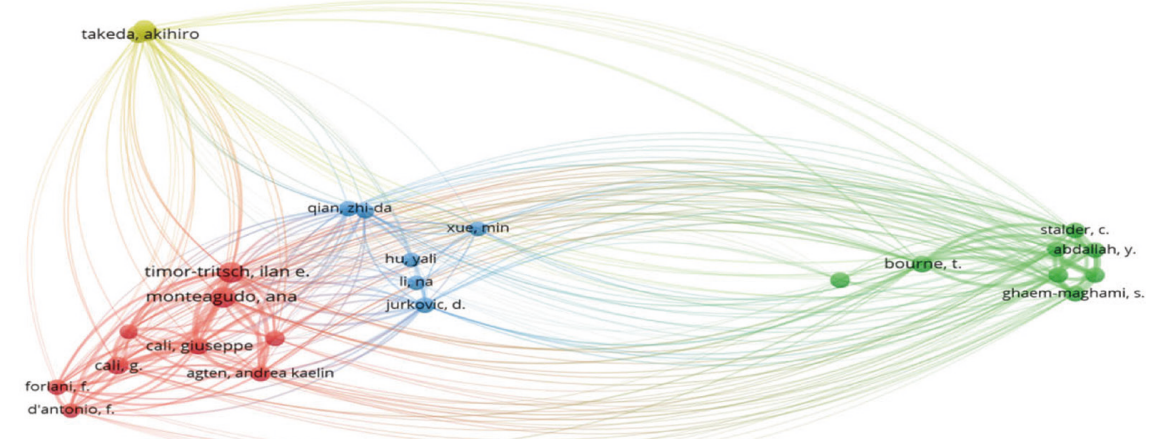

f

B

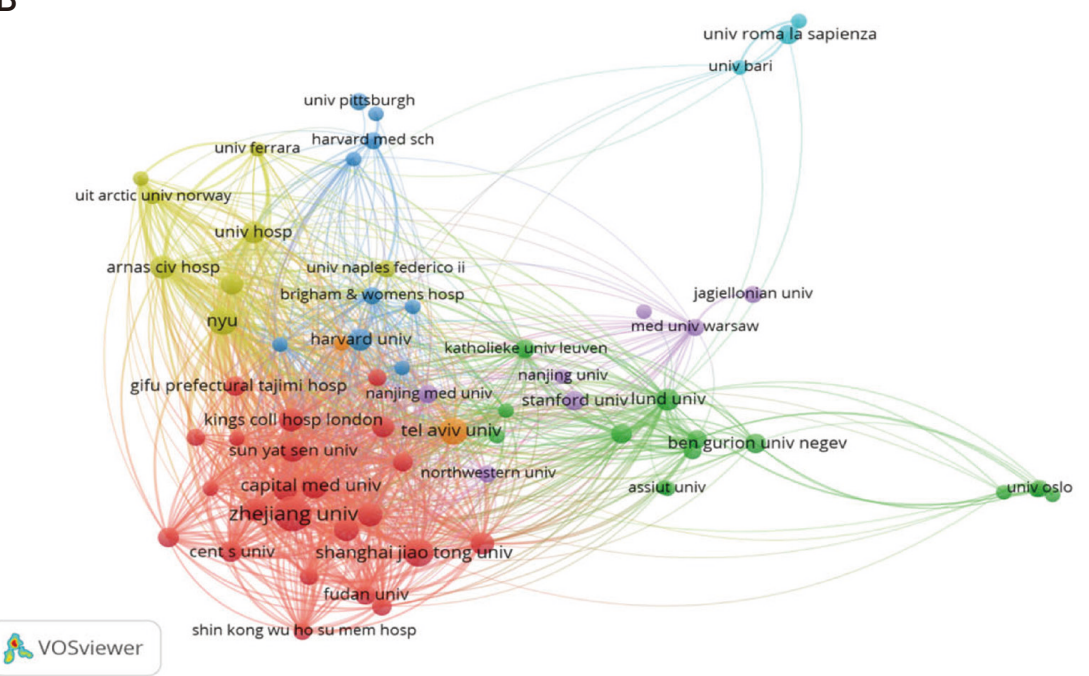

s vosviewer

C

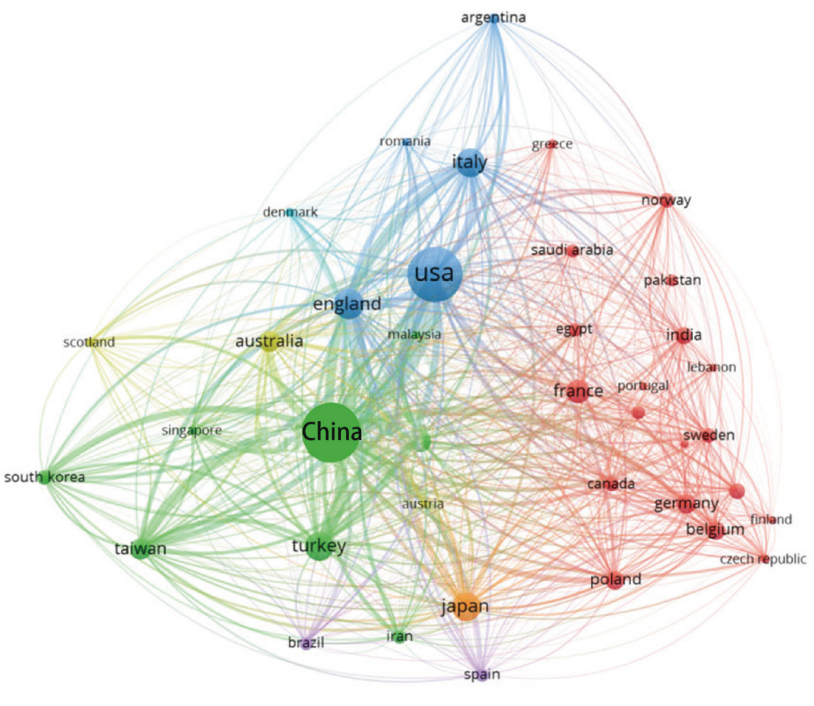

A vosviewer

Figure 4 Coupling analysis of CSP. (A) The author coupling analysis; (B) the institution coupling analysis; (C) the coupling analysis of countries or regions. The number of circles in the figure represents the number of analyzed items, and a larger circle corresponds to a greater contribution from the analyzed item. The line between the circles indicates the correlation between the analyzed items; a thicker or longer line corresponds to a greater correlation. CSP, cesarean scar pregnancy. 
in the research fields of CSP.

Analysis of research directions: the minimum number of occurrences of a keyword was set to 5 .

From the 3,764 keywords, 309 keywords reached the threshold and were divided into five clusters, which represent the global research direction of CSP in the last 20 years. The five clusters are "selection of the delivery mode with a scarred uterus after cesarean section" (Cluster 1, red), "risk factors" (Cluster 2, yellow), "diagnosis" (Cluster 3, blue), "treatment" (Cluster 4, purple), and "related basic and clinical experiments" (Cluster 5, green). In the study of "selection of the delivery mode with a scarred uterus after cesarean section", common keywords include "women, delivery, labor, birth, and uterine scar"; in the study of "risk factors", common keywords include "risk, rupture, prevalence, and defects"; in the study of "diagnosis", common keywords include "diagnosis, ectopic pregnancy, ultrasound, and ultrasonography"; in the study of "treatment", common keywords include "cesarean scar pregnancy, management, uterine artery embolization, and curettage"; and in the study of "related basic and clinical experiments", common keywords include "pregnancy, fertility, and repair" (Figure 5A,5B).

Research hotspot and developmental trend analysis: Through VOSviewer software, the keywords in the literature can be classified by color for the time of occurrence; accordingly, research hotspots can be represented by different colors (Figure 5C). The diagram shows that yellow and green nodes are emerging hotspots because the blue and purple nodes appear before the yellow and green nodes.

Therefore, the conclusion can be drawn that most studies may focus on HIFU to formulate consensus guidelines and so on in the future.

\section{Discussion}

\section{Research trends for CSP}

In this study, a combination of bibliometrics and visualized analysis was used to collate and analyze the data of 1,186 research papers on CSP published in 80 countries from 2001 to 2020. The data included countries and regions, institutions, authors, research directions, funding agencies, literature quality, etc. In addition, we also found some potential frontier research directions, which may provide convenience for future research.

Our results demonstrated that over the last couple of decades, although the number of publications descended in 2005, 2008, 2010 and 2016, it has shown an overall increasing trend and will continue growing in the future. Therefore, we can conclude that the study field of CSP will be hot in the future, probably because cesarean section has been increasing at an astounding rate due to the convenience for obstetricians and mothers, the fear of pain, the pressure of childbirth, concerns about genital modifications after vaginal delivery and so on (15-17).

\section{Global literature quality assessment}

As the most prominent contributors, China has published the most papers, accounting for approximately a quarter of the total, followed by the USA with a similar amount. On the other hand, among the top ten contributing countries or regions, publications of Taiwan were cited the most, followed by those from England, Israel and the USA, while China was second from the bottom. The top ten cited articles were from England, Taiwan, the USA, Italy, Sweden, and the Netherlands, and four of them were from the USA. Although China has conducted most studies, the quality of its outputs must be improved rapidly in the future. England and Taiwan had the highest quality literature, but their publications were too few to provide adequate references. Hence, through a comprehensive review of the quality and quantity of literature, the USA has undeniably played a crucial role in the study of CSP and has had a strong impact on academia related to CSP due to its close cooperation with other countries or regions, mainly because compared with other industrialized countries, the USA has a higher rate of cesarean section (18) and the most advanced medical and scientific study level.

The top ten institutions with the most publications were all from the top ten contributing countries or regions, illustrating that the establishment of first-rate institutions plays a crucial role in promoting the national academic research level. Among them, six were from China, and the others were from England, America, Israel and Italy. Zhejiang University from China has contributed the most papers, followed by NYU from the USA and Shanghai Jiao Tong University from China with the equal amount of publications.

However, the average number of citations per item of Chinese organizations were not sufficiently high; thus, their $\mathrm{H}$ indices were also relatively lower than those of organizations from other countries or regions, indicating that Chinese institutions should pursue high quality based 
A
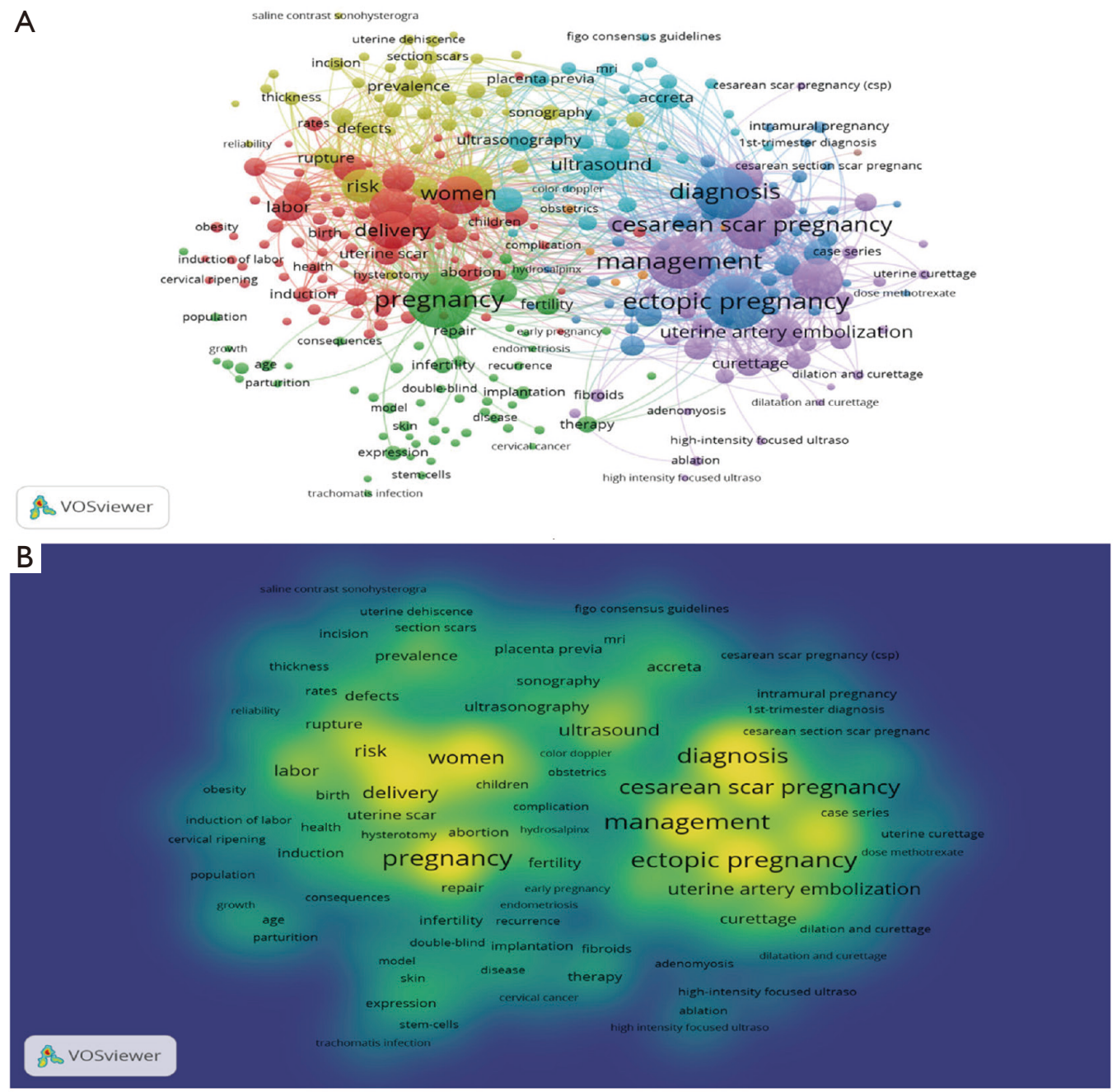

C

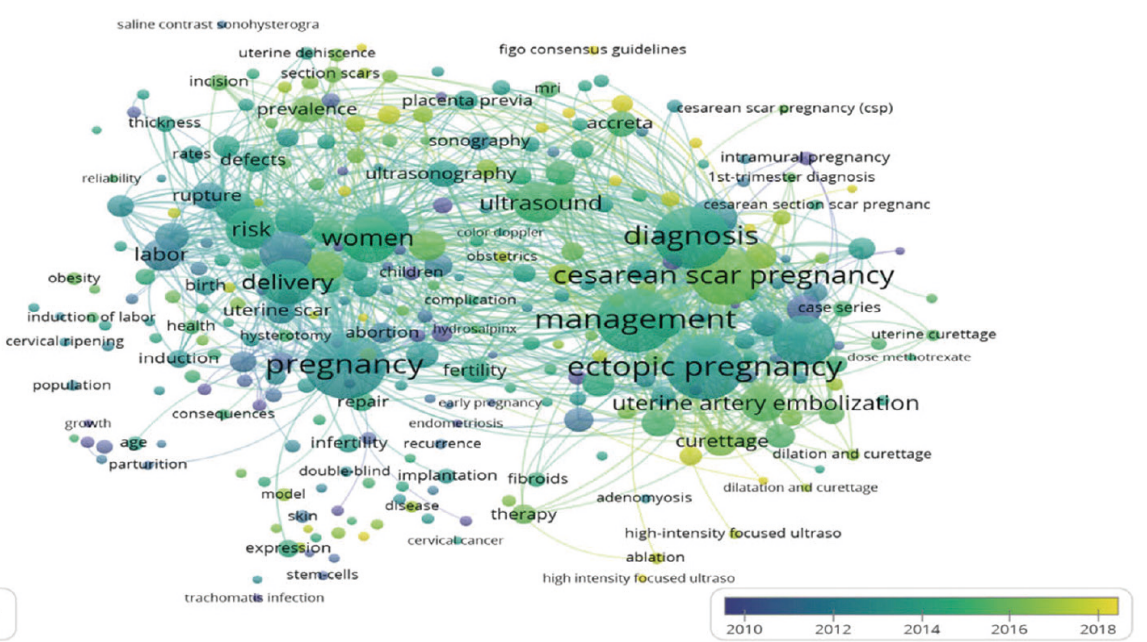

Figure 5 Co-occurrence analysis of CSP. (A) Mapping of keywords in the research area. The size of the dots represents the frequency of occurrence, and the keywords are divided into five clusters with different colors. (B) Heatmap of keywords according to the average frequency of occurrence. (C) Overlapping visualization diagram of color variation with the time axis [2010-2018]; yellow and red nodes appear later than blue and purple nodes, indicating that yellow nodes are hot keywords. CSP, cesarean scar pregnancy. 
on a large number of articles. NYU from the USA had the highest $\mathrm{H}$ index, with its number of publications and average citation frequency both ranking second. Kings Coll Hosp London from England had a dramatically high citation frequency, and an obvious gap was noted between him and next highest ranking author. Accordingly, we can conclude that NYU from the USA and King's College Hospital London from England are relatively mature in the research field of CSP and can be regarded as influential institutions for further learning and collaboration.

The top two related funding agencies were both from the USA, and four of the top ten funding agencies were also from the USA, which is one of the reasons why the leading authority on CSP research in the world is the USA. More importantly, adequate funding can provide support to ensure the development of scientific research and promote innovation.

Fournal of Obstetrics and Gynaecology Research has produced the most CSP-related papers over the last twenty years, indicating that it is a popular journal with researchers in the field of CSP. Nevertheless, its average citation frequency and $\mathrm{H}$ index were not sufficiently high to be the most influential journal in the field of CSP. Ultrasound in Obstetrics \& Gynecology, second to Fournal of Obstetrics and Gynaecology Research regarding publication outputs, has taken the lead for the average number of citations per item and $\mathrm{H}$ index. Four of the top ten cited articles were published, and the top two were both from this journal. Therefore, we can say that Ultrasound in Obstetrics \& Gynecology is generally considered the most authoritative journal in this research field. Meanwhile, of the top ten cited articles, the third, fourth and sixth were all published in American Fournal of Obstetrics and Gynecology, whose number of publications ranked third while its $\mathrm{H}$ index and average citation frequency both ranked second, showing that American Fournal of Obstetrics and Gynecology has also made significant and valuable contributions to studies on CSP. In the future, scholars should focus more on these journals to obtain access to international information and the latest developments in CSP.

Timor-Tritsch IE has conducted studies on CSP and written the most papers with the third highest citation frequency. Monteagudo A wrote only one fewer article than Timor-Tritsch IE. Articles published by him were cited the second most frequently. Jurkovic D may be the most influential scholar for the average number of citations per item, with his articles ranking first at a number much higher than those of Timor-Tritsch IE and Monteagudo A. One of his articles, "First-trimester diagnosis and management of pregnancies implanted into the lower uterine segment Cesarean section scar", published in Ultrasound in Obstetrics Gynecology in 2003, was among CSP-relevant papers with the highest citation frequencies, confirming that Jurkovic D has taken the lead in this research field, and focusing more on his study direction and achievements is beneficial for broadening our minds and promoting innovation.

Bibliographic coupling occurred when two articles referred to a shared third paper in their bibliographies, which was conducted to identify similarities between publications in terms of author, organization and country. Our data demonstrate that China and the USA have taken the lead in this field and have focused more on collaboration with other countries, indicating that placing more emphasis on academic exchange and cooperation can improve the academic level of a country. NYU from the USA was the most relevant institution. Timor-Tritsch IE, whose literature inspired the most scholars, is an authoritative leader in this research field.

\section{Analysis of research hotspots of CSP}

To grasp the directions of CSP research and current and future research hotspots, co-occurrence analysis of keywords is required. The analyzed keywords were divided into five clusters in different colors.

Cluster 1 (in red) concerned "selection of the delivery mode with a scarred uterus after cesarean section". Women with a history of cesarean section are at risk of uterine rupture, hemorrhea and even death in subsequent pregnancies. These women are advised to undergo an elective repeat cesarean delivery (ERCD) or a trial of labor after cesarean delivery (TOLAC) in the following pregnancy. ERCD effectively reduces the risk of massive bleeding due to complications of repeated cesarean sections, such as placenta previa and placenta implantation (19), which is recommended for those with a scarred uterus and a history of more than two cesarean sections (20). Except in extreme circumstances, this procedure must not be performed before 39 weeks (21) because of the high risk of respiratory distress in the newborn. TOLAC has been confirmed to contribute to reducing cesarean section (22). On the other hand, a sizable proportion of women who attempt TOLAC may end up with emergency cesarean section (23), which is associated with relatively higher maternal and neonatal morbidity than ERCD (24). This procedure is suitable for those with one previous 
low transverse uterine incision and a low likelihood of intrapartum uterine rupture. If obstetrical conditions permit, TOLAC has been acknowledged to be a better option (20). Practically, the preference of patients and clinicians, the timing of delivery, obstetrical history, and risks and benefits of TOLAC versus ERCD should be considered when addressing the question of which delivery mode is optimal.

Cluster 2 (in yellow) refers to "risk factors". Cesarean section, myomectomy and frequently induced abortions or induced abortions after cesarean delivery may injure the endometrium and myometrium, which may lead to one or more uterine incisions. Women who suffer from these conditions are more likely to have a CSP or recurrent CSP. According to reports $(9,25)$, maternal age, parity, hospital grade with respect to previous uterine surgeries, the interval between the last cesarean section and the current pregnancy, the number of induced abortions (especially after cesarean delivery), and the thickness of the lower uterine segment can act as risk factors for CSP by affecting uterine incision healing in multiple ways. Incomplete healing of the uterine incision may create scar defects where gestational sacs are more easily implanted and thus lead to various complications. The rate of cesarean scar defects in a random population of women with cesarean delivery was reported to reach $84 \%$ (26). Assessment of scar defects during cesarean section may help identify risk factors. Hence, further studies are crucial to identify risk factors for CSP and evaluate the relationship between CSP and cesarean scar defects.

Cluster 3 (in blue) can be summarized as "diagnosis". Without distinctive clinical symptoms, the diagnosis of a CSP is difficult to confirm in the initial stage. Especially when the gestational sac is located in the lower uterine segment, CSP, which may simply present vaginal bleeding with or without abdominal pain, is difficult to distinguish from early placenta accreta, threatened abortion, cervical pregnancy and other ectopic pregnancies (27-29). Benefiting from ultrasound imaging, CSP is easier to diagnose based on the history of cesarean delivery, visible clinical symptoms and imaging. The first discovery of a 7-week CSP through the use of transvaginal ultrasound occurred in 1990 (30). Subsequently, a growing number of case series have shown typical imaging of CSP, and the sensitivity of ultrasound for detecting CSP has been reported to be $84.6 \%$ (31). Thus, ultrasound, especially transabdominal and transvaginal sonography combined with color Doppler, has thus far been the optimum choice for the diagnosis of CSP because it can provide significant information and typing criteria, including the thickness of the scar, the position and size of the gestational sac and the situation of the fetal heart (32). Contrast-enhanced ultrasound, which was developed based on conventional ultrasound, is superior in sensitivity with respect to blood flow. A few studies stated that it can provide blood perfusion evidence for the gestational sac in real time and identify the site of embryo implantation more clearly, thus improving the accuracy of diagnosis $(33,34)$. Notably, however, diagnosis by the use of contrast-enhanced ultrasound has not been standardized, and scholars have not reached an agreement on the dose of microbubbles utilized in contrast-enhanced ultrasound (35). Magnetic resonance imaging (MRI) is also significant in CSP diagnosis. Because of higher soft-tissue contrast and better spatial resolution, the distance between the bladder, gestational sac and myometrium can be measured more accurately to precisely evaluate the degree of myometrial invasion as well as bladder involvement $(36,37)$. However, due to the lack of detection of the fetal heart and the time requirement, it is used less frequently than ultrasound. Regardless of the choice made, misdiagnosis or missed diagnosis of CSP may occur. Thus, improving the accuracy of CSP diagnosis, especially in the first trimester, has always been a research direction and hotspot attracting scholars worldwide.

Cluster 4 (in purple) involves "treatment". In the past, hysterectomy by emergency laparotomy may be performed to avoid uterine rupture and massive hemorrhage resulting from CSP. With research in this field, a multitude of management options for CSP have been proposed, including systematic injection of methotrexate (MTX), UAE, dilation and curettage, surgery and HIFU, but the optimal option remains to be determined. Although systemic injection of MTX was reported to be successful in treating CSP $(38,39)$, it was suggested to be dangerous in the treatment process and may not be as effective as in a regular ectopic pregnancy (40). One of the opinions against dilation and curettage is that this procedure may cause less thorough treatment and result in serious potential side effects, such as perforation of the uterus (28). A few scholars have confirmed that UAE may be useful by blocking blood flow at the implantation site and producing trophoblastic degeneration regardless of whether it is combined with other treatments $(41,42)$. Ultrasound-guided HIFU ablation has also been demonstrated to be noninvasive and effective. Fang et al. advised that UAE or HIFU can be used as a preoperative adjuvant therapy to reduce bleeding in operations and increase surgical safety (43). Thus, UAE, ablation, and HIFU with or without a combination of 
dilation and curettage may develop as emerging treatment directions, and scholars will devote themselves to exploring much safer and more effective therapies for CSP.

Cluster 5 (in green) concerns "related basic and clinical experiments". In light of the discovery that poor scar healing, adhesion caused by local inflammation, and treatments such as hysterectomy can be dramatically detrimental to the fertility of women (27), to facilitate scar repair and improve future fertility, research on the mechanism of a scarred uterus influencing fertility as well as the effects of various treatments on fertility have been conducted recently through basic animal and clinical experiments. Tumenjargal et al. (44) observed the fertility of 33 CSP patients who underwent dilation and curettage combined with UAE by writing letters or phoning to confirm whether they had become pregnant after treatment. Their studies showed a pregnancy rate of $44 \%$, indicating that UAE combined with dilation and curettage may be a safe and efficient management option for CSP. Lin et al. injected the fusion protein of CBD-VEGF into the scars of model rats with a scarred uterus to examine the effectiveness of CBD-VEGF in the repair of uterine scars and discovered that CBD-VEGF is involved in promoting remodeling of the scarred uterus and improving uterine function (45). According to the findings of experiments on rats, $\mathrm{Li}$ et al. indicated that chronic inflammation and a poor response to steroids in uterine scar tissues have adverse impacts on subsequent pregnancy (46). Xu et al.'s study showed that the stent/UC-MSC system could promote collagen degradation in uterine scars by upregulating MMP-9 in rat models to facilitate reestablishment of the myometrium, endometrium and blood vessels in uterine scars (47). Although few studies in this field have been conducted, such findings are of great importance in CSP research.

Figure $5 C$ is a superimposed visual diagram, which is of great significance when discussing research directions. Coloring closer to purple corresponds to an earlier research direction, and coloring closer to yellow corresponds to a more recent research direction. As shown in the figure, the yellow and green keywords were mostly distributed in Cluster 2, Cluster 3 and Cluster 4, indicating that vast space remains for exploration of the diagnosis, treatment and risk factors for CSP, and HIFU and the formulation of consensus guidelines are the current research hotspots in this field.

As a novel noninvasive treatment, HIFU can cause a sharp temperature increase up to $60-100{ }^{\circ} \mathrm{C}$ by percutaneous means to induce necrosis and fibrosis of the fetal tissue, which avoids the risks associated with laparotomy or laparoscopic surgery and achieves a high success rate in the treatment of CSP (48). It can act directly on the embryo without affecting the surrounding normal tissues, is superior to UAE in reducing the risk of recurrence of CSP (49), and is presumably a priority option for early treatment of CSP, but it still needs to be validated in a large, multicenter randomized controlled trial (50). On the other hand, HIFU has difficulty achieving satisfactory efficacy for patients with larger gestational sacs, higher HCG levels or sufficient blood supply around the gestational sac (43), and postoperative complications such as fever, hematuria, pelvic pain, skin burn, nerve or intestinal injury and lower limb pain have also been observed (51,52). At present, CSP is difficult to diagnose in time, and the optimal treatment is still uncertain. Although the Chinese Medical Association (CMA) issued the Expert Consensus in 2016 and the Society for Maternal-fetal Medicine (SMFM) issued the Consult in 2020, no relevant international and national clinical guidelines have been issued thus far. Therefore, how to make better use of HIFU technology and establish standardized clinical guidelines as soon as possible is the future research direction of obstetricians and gynecologists.

Although the study provides the current research status and hotspots of CSP, some limitations remain. First, the study was limited to years before 2020, and newly published literature was not included. Due to the short publication time, some high-quality papers published recently may be affected by the total citation frequency and $\mathrm{H}$ index, leading to errors in the overall quality evaluation. Second, our literature search was limited to the WOS database and restricted to published articles only, and some literature may have been omitted. Finally, this study was limited to English literature, which may lead to certain bias in statistical results.

In conclusion, research and publications on CSP have shown considerable growth in the past few decades and will continue to develop in the future. The United States maintains a leading position in this field. All countries need to strengthen their efforts to build first-class institutions, enhance academic cooperation and improve the quality of research starting from CSP research hotspots to improve the overall diagnostic rate, identify the best treatment options, and gradually develop global guidelines.

\section{Acknowledgments}

Funding: This work was supported by Guangdong 
Provincial Traditional Chinese Medicine Heritage Studio to Gaopi Deng [Letter of Guangdong Traditional Chinese Medicine (2019) No. 5]; Traditional Chinese Medicine of the Bureau of Guangdong Province (No. 20211120); and China Maternal and Child Health Association - China Resources Sanjiu Special Research Fund for Maternal and Child Health of Integrated Traditional Chinese and Western Medicine (No. ZXY2020B02).

\section{Footnote}

Conflicts of Interest: All authors have completed the ICMJE uniform disclosure form (available at https://apm. amegroups.com/article/view/10.21037/apm-21-3976/coif). The authors have no conflicts of interest to declare.

Ethical Statement: The authors are accountable for all aspects of the work in ensuring that questions related to the accuracy or integrity of any part of the work are appropriately investigated and resolved.

Open Access Statement: This is an Open Access article distributed in accordance with the Creative Commons Attribution-NonCommercial-NoDerivs 4.0 International License (CC BY-NC-ND 4.0), which permits the noncommercial replication and distribution of the article with the strict proviso that no changes or edits are made and the original work is properly cited (including links to both the formal publication through the relevant DOI and the license). See: https://creativecommons.org/licenses/by-nc$\mathrm{nd} / 4.0 /$.

\section{References}

1. López-Girón MC, Nieto-Calvache AJ, Quintero JC, et al. Cesarean scar pregnancy, the importance of immediate treatment. J Matern Fetal Neonatal Med 2020. [Epub ahead of print]. doi: 10.1080/14767058.2020.1742691.

2. Birch Petersen K, Hoffmann E, Rifbjerg Larsen C, et al. Cesarean scar pregnancy: a systematic review of treatment studies. Fertil Steril 2016;105:958-67.

3. Botden S, Strijkers R, Fransen S, et al. The use of curved vs. straight instruments in single port access surgery, on standardized box trainer tasks. Surg Endosc 2011;25:2703-10.

4. Seow KM, Huang LW, Lin YH, et al. Cesarean scar pregnancy: issues in management. Ultrasound Obstet Gynecol 2004;23:247-53.
5. Calì G, Timor-Tritsch IE, Palacios-Jaraquemada J, et al. Outcome of Cesarean scar pregnancy managed expectantly: systematic review and meta-analysis. Ultrasound Obstet Gynecol 2018;51:169-75.

6. Li HT, Luo S, Trasande L, et al. Geographic Variations and Temporal Trends in Cesarean Delivery Rates in China, 2008-2014. JAMA 2017;317:69-76.

7. Liang J, Mu Y, Li X, et al. Relaxation of the one child policy and trends in caesarean section rates and birth outcomes in China between 2012 and 2016: observational study of nearly seven million health facility births. BMJ 2018;360:k817.

8. Hoffman T, Lin J. Cesarean Scar Ectopic Pregnancy: Diagnosis With Ultrasound. Clin Pract Cases Emerg Med 2020;4:65-8.

9. Zhou X, Li H, Fu X. Identifying possible risk factors for cesarean scar pregnancy based on a retrospective study of 291 cases. J Obstet Gynaecol Res 2020;46:272-8.

10. Pu QH, Lyu QJ, Su HY. Bibliometric analysis of scientific publications in transplantation journals from Mainland China, Japan, South Korea and Taiwan between 2006 and 2015. BMJ Open 2016;6:e011623.

11. Xing D, Zhao Y, Dong S, et al. Global research trends in stem cells for osteoarthritis: a bibliometric and visualized study. Int J Rheum Dis 2018;21:1372-84.

12. Bornmann L, Leydesdorff L. Scientometrics in a changing research landscape: bibliometrics has become an integral part of research quality evaluation and has been changing the practice of research. EMBO Rep 2014;15:1228-32.

13. Zhang $Y$, Zhang T, Liu X, et al. Research trends of pregnancy with scarred uterus after cesarean: a bibliometric analysis from 1999 to 2018. J Matern Fetal Neonatal Med 2020. [Epub ahead of print]. doi: 10.1080/14767058.2020.1830368.

14. Bertoli-Barsotti L, Lando T. A theoretical model of the relationship between the h-index and other simple citation indicators. Scientometrics 2017;111:1415-48.

15. Betrán AP, Ye J, Moller AB, et al. The Increasing Trend in Caesarean Section Rates: Global, Regional and National Estimates: 1990-2014. PLoS One 2016;11:e0148343.

16. Torloni MR, Betrán AP, Montilla $\mathrm{P}$, et al. Do Italian women prefer cesarean section? Results from a survey on mode of delivery preferences. BMC Pregnancy Childbirth 2013;13:78.

17. Hellerstein S, Feldman S, Duan T. China's $50 \%$ caesarean delivery rate: is it too high? BJOG 2015;122:160-4.

18. Menacker F, Hamilton BE. Recent trends in cesarean delivery in the United States. NCHS Data Brief 
2010;(35):1-8.

19. Clark SL, Koonings PP, Phelan JP. Placenta previa/accreta and prior cesarean section. Obstet Gynecol 1985;66:89-92.

20. Sentilhes L, Vayssière C, Beucher G, et al. Delivery for women with a previous cesarean: guidelines for clinical practice from the French College of Gynecologists and Obstetricians (CNGOF). Eur J Obstet Gynecol Reprod Biol 2013;170:25-32.

21. Tita AT, Landon MB, Spong CY, et al. Timing of elective repeat cesarean delivery at term and neonatal outcomes. $\mathrm{N}$ Engl J Med 2009;360:111-20.

22. Sabol B, Denman MA, Guise JM. Vaginal birth after cesarean: an effective method to reduce cesarean. Clin Obstet Gynecol 2015;58:309-19.

23. Guise JM, Eden K, Emeis C, et al. Vaginal birth after cesarean: new insights. Evid Rep Technol Assess (Full Rep) 2010;(191):1-397.

24. Gilbert SA, Grobman WA, Landon MB, et al. Elective repeat cesarean delivery compared with spontaneous trial of labor after a prior cesarean delivery: a propensity score analysis. Am J Obstet Gynecol 2012;206:311.e1-9.

25. Luo L, Ruan X, Li C, et al. Early clinical features and risk factors for cesarean scar pregnancy: a retrospective casecontrol study. Gynecol Endocrinol 2019;35:337-41.

26. OuYang Z, Yin Q, Xu Y, et al. Heterotopic cesarean scar pregnancy: diagnosis, treatment, and prognosis. $\mathrm{J}$ Ultrasound Med 2014;33:1533-7.

27. Maymon R, Svirsky R, Smorgick N, et al. Fertility performance and obstetric outcomes among women with previous cesarean scar pregnancy. J Ultrasound Med 2011;30:1179-84.

28. Osborn DA, Williams TR, Craig BM. Cesarean scar pregnancy: sonographic and magnetic resonance imaging findings, complications, and treatment. J Ultrasound Med 2012;31:1449-56.

29. Timor-Tritsch IE, Monteagudo A, Cali G, et al. Cesarean scar pregnancy and early placenta accreta share common histology. Ultrasound Obstet Gynecol 2014;43:383-95.

30. Rempen A, Albert P. Diagnosis and therapy of an in the cesarean section scar implanted early pregnancy. Z Geburtshilfe Perinatol 1990;194:46-8.

31. Rotas MA, Haberman S, Levgur M. Cesarean scar ectopic pregnancies: etiology, diagnosis, and management. Obstet Gynecol 2006;107:1373-81.

32. Timor-Tritsch IE, Monteagudo A, Santos R, et al. The diagnosis, treatment, and follow-up of cesarean scar pregnancy. Am J Obstet Gynecol 2012;207:44.e1-13.

33. Li HD, Zhang ZK, Yang H, et al. The Clinical Application of Intravenous Contrast-enhanced Ultrasound in Uterine Cesarean Scar Pregnancy. Journal of International Obstetrics and Gynecology 2015;42:355-7.

34. Xiong X, Yan P, Gao C, et al. The Value of ContrastEnhanced Ultrasound in the Diagnosis of Cesarean Scar Pregnancy. Biomed Res Int 2016;2016:4762785.

35. Liu D, Yang M, Wu Q. Application of ultrasonography in the diagnosis and treatment of cesarean scar pregnancy. Clin Chim Acta 2018;486:291-7.

36. Peng KW, Lei Z, Xiao TH, et al. First trimester caesarean scar ectopic pregnancy evaluation using MRI. Clin Radiol 2014;69:123-9.

37. Singh N, Tripathi R, Mala YM, et al. Comparison of scar thickness measurements using trans-vaginal sonography and MRI in cases of pregnancy with previous caesarean section. Do they correlate with actual scar thickness? J Obstet Gynaecol 2013;33:810-3.

38. Jurkovic D, Hillaby K, Woelfer B, et al. First-trimester diagnosis and management of pregnancies implanted into the lower uterine segment Cesarean section scar. Ultrasound Obstet Gynecol 2003;21:220-7.

39. Haimov-Kochman R, Sciaky-Tamir Y, Yanai N, et al. Conservative management of two ectopic pregnancies implanted in previous uterine scars. Ultrasound Obstet Gynecol 2002;19:616-9.

40. Kanat-Pektas M, Bodur S, Dundar O, et al. Systematic review: What is the best first-line approach for cesarean section ectopic pregnancy? Taiwan J Obstet Gynecol 2016;55:263-9.

41. Yang XY, Yu H, Li KM, et al. Uterine artery embolisation combined with local methotrexate for treatment of caesarean scar pregnancy. BJOG 2010;117:990-6.

42. Rizk B, Holliday CP, Owens S, et al. Cervical and Cesarean scar ectopic pregnancies: Diagnosis and management. Middle East Fertility Society Journal 2013;18:67-73.

43. Fang S, Zhang P, Zhu Y, et al. A Retrospective Analysis of the Treatment of Cesarean Scar Pregnancy by High-Intensity Focused Ultrasound, Uterine Artery Embolization and Surgery. Front Surg 2020;7:23.

44. Tumenjargal A, Tokue H, Kishi H, et al. Uterine Artery Embolization Combined with Dilation and Curettage for the Treatment of Cesarean Scar Pregnancy: Efficacy and Future Fertility. Cardiovasc Intervent Radiol 2018;41:1165-73.

45. Lin N, Li X, Song T, et al. The effect of collagen-binding vascular endothelial growth factor on the remodeling of scarred rat uterus following full-thickness injury. Biomaterials 2012;33:1801-7. 
46. Li Z, Bian X, Ma Y, et al. Uterine Scarring Leads to Adverse Pregnant Consequences by Impairing the Endometrium Response to Steroids. Endocrinology 2020;161:bqaa174.

47. Xu L, Ding L, Wang L, et al. Umbilical cord-derived mesenchymal stem cells on scaffolds facilitate collagen degradation via upregulation of MMP-9 in rat uterine scars. Stem Cell Res Ther 2017;8:84.

48. Huang L, Du Y, Zhao C. High-intensity focused ultrasound combined with dilatation and curettage for Cesarean scar pregnancy. Ultrasound Obstet Gynecol 2014;43:98-101.

49. Chen L, Xiao S, Zhu X, et al. Analysis of the Reproductive Outcome of Patients with Cesarean Scar Pregnancy Treated by High-Intensity Focused Ultrasound and

Cite this article as: Qiu P, Lin X, Li A, Deng G. Research status and hotspots of cesarean scar pregnancy from 2001 to 2020: a bibliometric analysis. Ann Palliat Med 2022;11(2):631-646. doi: 10.21037/apm-21-3976
Uterine Artery Embolization: A Retrospective Cohort Study. J Minim Invasive Gynecol 2019;26:883-90.

50. Xiao X, Feng Z, Li T, et al. Comparing the Efficacy and Safety of High-Intensity Focused Ultrasound and Uterine Artery Embolization in Caesarean Scar Pregnancy: A Meta-analysis. Adv Ther 2019;36:1314-25.

51. Shaw CJ, ter Haar GR, Rivens IH, et al. Pathophysiological mechanisms of high-intensity focused ultrasound-mediated vascular occlusion and relevance to non-invasive fetal surgery. J R Soc Interface 2014;11:20140029.

52. Hong Y, Guo Q, Pu Y, et al. Outcome of high-intensity focused ultrasound and uterine artery embolization in the treatment and management of cesarean scar pregnancy: A retrospective study. Medicine (Baltimore) 2017;96:e7687. 